Original Research Paper

\title{
Investigating QoS and Performance of Received Signal Strength Indicator in Fiber Optics Broadband Data Communication
}

\author{
Owusu Nyarko-Boateng, Adebayo F. Adekoya and Benjamin A. Weyori \\ Department of Computer Science, the University of Energy and Natural Resources, Sunyani, Ghana
}

\author{
Article history \\ Received: 20-05-2019 \\ Revised: 26-06-2019 \\ Accepted: 01-08-2019 \\ Corresponding Author: \\ Owusu Nyarko-Boateng \\ Department of Computer \\ Science, the University of \\ Energy and Natural Resources, \\ Sunyani, Ghana \\ Email: owusu.nyarko-boateng@uenr.edu.gh
}

\begin{abstract}
There has been an unusual drop of Fiber Optics Broadband (FOB) internet speed in certain localities in Ghana which severely affect users' experience. This phenomenon have had a serious impact on the quality of service of internet delivery. This paper accessed the quality service of FOB wireless signal between the last mile cell site and the FOB wireless Access Point (AP). The essential components that the paper looked at were accessibility, availability and usability of FOB wireless signal which defines the QoS of a robust internet connection. This paper investigated the quality of FOB wireless signal and how lower antenna gain could affect the received signal strength of the FOB wireless AP. The study adopted three regulatory licensed indicators-Data Access Success Rate, Data Drop Rate and Data Throughput-in measuring accessibility, availability and usability of the received signal strength. A drive-test was conducted in five cities in Ghana where FOB services were available to assess the performance of the FOB wireless signal at peak and off-peak period. It was observed that the strength of the signal reduced significantly during peak hours; this phenomenon severely affected the QoS of the internet. Kalma's filter and other communication techniques were used to analyze the results of the drive-test. The paper also provided conceptual framework which included suggestions and recommendations on how the Mobile Network Operators (MNOs) could adopt new measures to improve and upgrade their communication infrastructure, to ensure users always have the most efficient and quality of internet service delivery through FOB.
\end{abstract}

Keywords: Quality of Service, Data Access Success Rate, Data Drop Rate and Data Throughput, RSSI, Signal Loss

\section{Introduction}

In mobile data communication services, it is strongly expected that industry players such as the MNOs, provide quality services to its subscribers as in the case of FOB internet services. In most cases, the MNOs are not able to meet these targets of ensuring subscribers are given efficient and quality data services (Varma, 2015). Poor quality of service due to attenuated signal is usually recorded at peak hours in FOB data (internet) service delivery. The major problem this paper seeks to investigate and provide solutions to include FOB wireless signal loss, packet loss, error bit rate, poor throughput, transmission delay, service unavailability, jitter, latency, limited infrastructure vis-à-vis a large number of subscribers, which mostly result in high-level of congestion. This paper investigated the quality of data services of FOB (Ahmadi, 2011) in peak and off-peak hours. Three adopted regulatory license indicators were assessed during this investigation and assessment. These regulatory license indicators included Data Access Success Rate, Data Drop Rate and Data Throughput. The paper also sought the views of users and their perception on the quality of service delivery at the target hours. The research also provided suggestions and recommendations on how the MNOs could improve and upgrade their communication infrastructure (Nyarko-Boateng and Adekoya, 2019), to ensure the users always have the most efficient and quality of internet service delivery (Bhatt et al., 2019). The paper further performs a comparative analysis of the impact of these three regulatory licenses on QoS FOB service. Kar and Sanyal (2017) established that the quality of service is an important indicator used in determining the efficiency of 
MNOs in terms of services rendered. In FOB internet services, availability, accessibility and retainable are the three key factors used in evaluating the regulatory license of an operator. The questions this research tried to answer were (i) is the MNO providing the user with the broadband speed they subscribed to? (ii) Does the user's broadband speeds vary at different hours of the day? What causes these variations if there are? Users' of the service expect maximum satisfaction for the services they have paid for. The maximum satisfaction factor has now become a difficult task to achieve especially in the telecommunication industry; some major reasons which can be attributed to this are the mismatch of a hardware component, expansion in customer base and infrastructural expansion and the severe effect on the RSSI (Bhatt et al., 2019).

\section{Related Work}

Data communication has become a major driver of economic growth in the world today. QoS of this essential driver must be ensured at all times to enhance internal and external communications in organizations, government agencies, the military and so on. Data communications is basically the process of using computing and communication technologies to transfer data from source to a destination and vice versa (Karn et al., 2017). It enables the movement of digital data between two or more communication nodes, regardless of geographical location, technological medium or data contents (Liu et al., 2012).

Fiber optic broadband typically deploys fiber optic cables to send data. It is very fast, especially compared to traditional Asymmetric Digital Subscriber Line (ADSL) broadband (Bhatt et al., 2019). ADSL broadband is a connection provided over home telephone lines. According to Karn et al. (2017), the FOB connection is generally made up of two main network infrastructure, thus, fiber optic cables terminated at local street cabinet, which then connects to user's home via copper phone lines. Secondly, service providers offer broadband via wireless medium from the last mile cell site to the user's wireless AP (Karn et al., 2017).

As asserted by Hadi et al. (2019), the cable and wireless FOB connections provides several advantages and disadvantages over each other. One of the major disadvantages of the wireless FOB is the attenuation of the transmitted signal over a certain distance. In most times within certain periods of the day; that is either peak or offpeak hours, the FOB wireless signal attenuate significantly. Several methods have been used to evaluate the performance of the FOB signal which includes the efficiency of Analog Radio over Fiber (A-RoF) and Digital Radio over Fiber (D-RoF) by Hadi et al. (2019).

This paper presents an investigation into the weak FOB signal during the peak and off-peak hours and then propose a framework to mitigate the impairments present in the FOB wireless connections. In order to improve the quality of received signal Kalman's filter were used by Belgacem and Lamir (2018) and Khan et al. (2014) to optimize wireless network between local node to a wireless AP.

\section{Data Communication Technologies}

In the last three decades, there has been a resounding growth in data transfer rate, especially from technologies like GPRS, EDGE, 3G, 4G and now 5G. These technologies offered different data transfer rate and improved bandwidth (Bhatt et al., 2019). The quality of service delivery by these technologies depended largely on the kind of network element and infrastructure deployed, which is characterized by having a defined range of speed (Varma, 2015).

General Packet Radio Service (GPRS) is a packetbased wireless communication service which fall within the $2 \mathrm{G}$ technolog; GPRS support a download speed of up to $114 \mathrm{Kbps}$. One of the major limitations of GPRS was that GPRS data could not be sent while a voice call was in progress. GPRS technology was later improved to an Enhanced Data GSM Evolution (EDGE). EDGE and GPRS were both $2 \mathrm{G}$ technology, but EDGE was significantly faster with a download speed of up to $384 \mathrm{Kbps}$. EDGE was often referred to as $2.5 \mathrm{G}$ network as it also has some characteristics of a $3 \mathrm{G}$ network, but it did not satisfy the specification. Introduction of the 3G/Universal Mobile Telecommunication Service (UMTS) technology made video calling and seamless streaming of video possible. The technology came with a laudable download speed of up to $3.1 \mathrm{Mbps} .3 \mathrm{G} / \mathrm{UMTS}$ allows a download/upload speed of between $384 \mathrm{Kbps}$ to $2 \mathrm{Mbps}$. In Code-Division Multiple Access (CDMA) technologies, the $3 \mathrm{G}$ network is referred to as Evolution-Data Optimized (EvDO) which offers similar maximum upload speed as UMTS for GSM data communications (Boateng et al., 2019). HighSpeed Packet Access (HSPA) technology is an enhancement to $3 \mathrm{G}$. However, it has a faster upload and download speed than $3 \mathrm{G}$.

The maximum speed can scale as high as $14 \mathrm{Mbps}$. HSPA is sometimes called $3.5 \mathrm{G}$. It is also important to note that both HSDPA and HSUPA belongs to the HSPA family. While HSDPA stands for High-Speed Downlink Packet Access, HSUPA means High-Speed Up-link Packet Access. Evolved High-Speed Packet Access (HSPA+) is an evolution of HSPA (HSDPA and HSUPA). These are $4 \mathrm{G}$ technologies that allows download at a rate of up to $168 \mathrm{Mbps}$. 4G Long Term Evolution (LTE) is a $4 \mathrm{G}$ communication standard that supports HD video streaming, download speed as high as 299.6 Mbps. Fiber optics broadband has the same speed as $4 \mathrm{G} / \mathrm{LTE}$ provides. LTE-A/5G is the most recent mobile data communication technologies that make both uploads and downloads faster at $1 \mathrm{~GB} / \mathrm{s}$ and a latency of less than 1ms (Shaddad et al., 2012). 


\section{Fiber Optics Network}

High bandwidth delivery and reliable internet access have been on high demand in the industries, homes and office for efficient and reliable communications. The consideration for an accessible and flexible Passive Optical Network (PON) ensures that fiber optics networks are extended to the user via several infrastructure and channels. This phenomenon has its own challenges since service delivery is quite expensive and fiber cable is prone to destruction. The large scale fiber optics network infrastructure are transmitted over a long distance from the MNOs switching centre to the last mile. There are several types of last mile fiber connections that MNOs deploys, each one varying in how the architectural design has been used for the FOB internet connection. Each architectural design is referred to as Fiber to the X (FTTx), where $\mathrm{x}$ represents the location where the optical fiber connection is finally terminated. This infrastructure form part of the MNOs protected links within cities and towns, where optical distribution parch panels are placed at the telecommunication mast to distribute FTTX services to the appropriate destination (Ahmadi, 2011).

\section{Fttp/Ftth/Fttb/Fttd}

Fiber to the premise, home, business or desktop are well known PON last mile distributed fiber lines. This network infrastructure provides FOB service direct to the premises of the user. These are also the most expensive fiber optics layout by the MNOs.

\section{FTTB}

The deployment of fiber to the building provides enhanced network scalability where the fiber services are distributed throughout the building by copper lines. This is a popular choice for apartment buildings, hotels, schools, churches or buildings that provide Internet to several other businesses and organizations.

\section{Fttc/Fttn/Ftts}

Fiber to the cabinet, neighbourhood, or street are the most used fiber optics network infrastructure. These form of fiber network infrastructure are deployed to the street cabinets, through a single mode fiber optics cable and then distributed by copper cables to the premises of users. This is the most affordable fiber optic internet connection by the MNOs because the MNOs do not have to invest in costly infrastructure to the separate premises and it can be re-distributed when new residence or businesses request for such services (Bhatt et al., 2019).

\section{Fiber Optics Broadband Technology}

FOB uses fiber optic wireless technology to deliver data services to the homes and businesses of users. FOB is usually a wireless internet service which can be accessed from the wireless router as an Access Point (AP). The AP receives the wireless broadband signal from the wireless gateway which is the terminal node of the fiber optics networks; this covers the radius of between $1-3 \mathrm{~km}$ range. The AP then broadcast the services to the user equipment using the wireless fidelity (Wi-Fi) standards. The existing WiFi standard such as IEEE $802.11 \mathrm{a} / \mathrm{b} / \mathrm{g} / \mathrm{n}$ which supports the data rate of $54 / 11 / 54 / 300 \mathrm{Mb} / \mathrm{s}$ respectively in a range of $100 \mathrm{~m}$ is used (Ahmadi, 2011).

However, besides the challenges in the deployment of FTTx services, there is no flexibility in the terminal node communication to the wireless AP at the user's premises. This situation called for the deployment of wireless communication system known as the FOB to ensure users within the coverage radius of the broadband signal have flexibility in accessing FOB services (Ghassemlooy et al., 2017). Figure 1 shows the complete architectural design of FOB network infrastructure. There are several fiber optics communication components within the layout, which enhances the speed, bandwidth, data integrity and quality of service.

MNO switching centres receive the main submarine fiber optics cable which gateway to the international traffic termination linking countries and continents through submarine transmission system (Ahmadi, 2011). The Central Office (CO) are deployed with optical splitting components and other data traffic management device such as DWDM (Densed wavelength division multiplexing), FIB (Fiber interface board), Optical Distribution Frame (ODF), etc. These connections are usually long distance, which requires single mode fiber optics cable. Long haul fiber optics cable and distributed fiber cable, transmits data from the various deployment centres to the last mile known as the wireless gateway, where the FOB service is deployed for easy accessibility to users' equipment through the FOB wireless router (Ghassemlooy et al., 2017).

User's bandwidth subscription is expected to be provided by the MNOs through the FOB networks. For instance, if a user subscribes to a data bandwidth of $10 \mathrm{~Gb}$ per month, MNO must ensure this value is been received at the user's end at all times. Unfortunately, this bandwidth (Kakati and Arya, 2018) usually drops or flaps in the course of transmission during specific periods. $10 \mathrm{~Gb}$ may be recorded at the wireless gateway, but the handover process usually degrades the quality of the FOB wireless signal, which affects the received signal at the user's premises (Ahmadi, 2011). The research conducted a drive-test to examine the Received Signal Strength Indicators (RSSI), which defines the three adopted key licensed indicators of the broadband signal. 


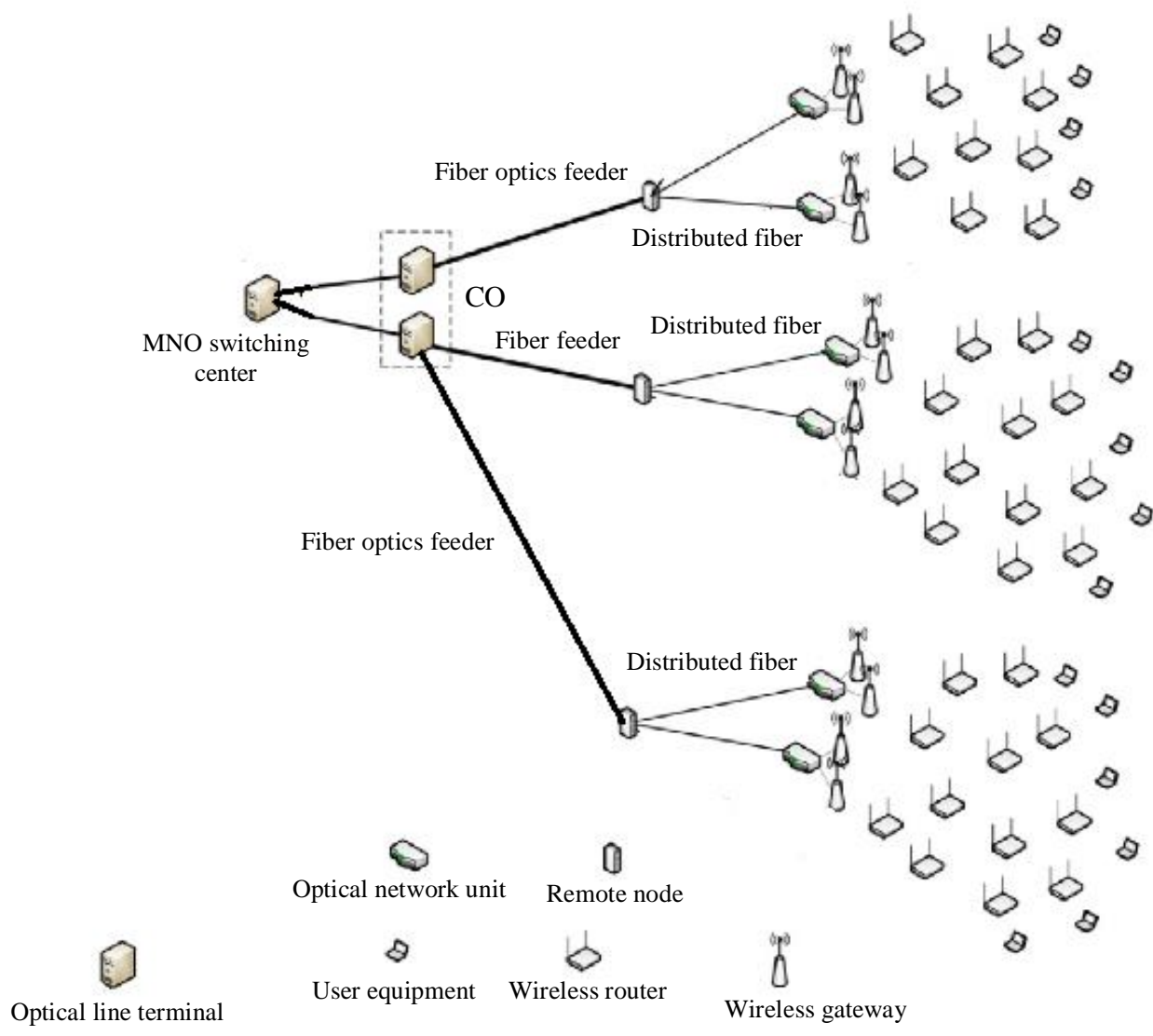

Fig. 1: Fiber Optics, broadband network infrastructure

Table 1: Indicators used to determine QoS in Ghana

\begin{tabular}{llll}
\hline Indicator & $\begin{array}{l}\text { Data access } \\
\text { success rate }(\%)\end{array}$ & $\begin{array}{l}\text { Data drop } \\
\text { rate }(\%)\end{array}$ & $\begin{array}{l}\text { Throughput } \\
(\mathrm{Mbps})\end{array}$ \\
\hline Threshold & $(>$ or $=95 \%)$ & $(<5 \mathrm{sec})$ & $(>$ or $=1 \mathrm{Mbps})$ \\
\hline
\end{tabular}

\section{Indicators Used to Determine QoS in Ghana}

The three key licensed indicators used in measuring the quality of data delivery in Ghana are indicated in Table 1 . The same indicators have also been used to assess FOB internet quality of service. This suggests MNOs are tasked to ensure quality service delivery based on the data access success rate, data drop rate and the data throughput. In most case, users often complain of poor data service; others say there are drops of service quite often and delay in surfing the internet (Karn et al., 2017). This situation prompted the use of drive-test technique to assess the quality of FOB service delivered by the MNOs.

\section{Data Access Success Rate}

Data Access Success Rate (DASR) is the probability of success in connecting to the public server. DASR must be greater than or equal to 95 per cent $(\geq 95 \%)$ :

$$
\begin{aligned}
& \operatorname{DASR}(\%)= \\
& \frac{\text { Total number of successful PDP content Activation }}{\text { Total number of PDP content Activation request }}
\end{aligned}
$$

\section{Data Drop Rate}

Data Drop Rate is the probability of drop in connection to a public server without end user's intervention. DDR must be less than or equal to one per cent $(\leq 1 \%)$ :

$$
\begin{aligned}
& \operatorname{DDR}(\%)= \\
& \frac{\text { Total umber of Aborted PDPContent Activation }}{\text { Total number of PDPcontent Activation request }} \times 100 \%
\end{aligned}
$$

\section{Data Throughput}

Data Throughput is the rate of data transfer. As per the $4 \mathrm{G}$ and fiber optics license obligations, the minimum data transfer rate for $90 \%$ of data connections should be $1 \mathrm{Mbps}$ or better ( $\geq 1 \mathrm{Mbps}$ ). 


\section{Methodology}

The aim of this research was to measure the QoS and performance of FOB wireless signal. This measurement was achieved by assessing the signal strength between the wireless gateway and the AP. A comprehensive drive-test was conducted in some selected cities in Ghana, where FOB services were operational. The test focused mainly on measuring values of the licensed indicators. At the time of conducting this test, only two MNOs in Ghana were providing FOB internet service at some part of the country. The names of these two MNOs have been changed to Net1 and Net2 for the sake of anonymity. The drive-test result was tabulated, as shown in Table 2 and Table 3. The drive-tests were conducted at both peak and off-peak time within the specified period. Off-peak time was between the hours of 10:00GMT 14:00 GMT and the peak hours were between 18:00GMT - 20:00 GMT. In all these five locations the test was done at a reference distance of $1.5 \mathrm{~km}$ from the last mile cell site.

\section{Drive-Test Result and Analysis}

The results of the tests for both peak and off-peak hours were tabulated in Table 2 and 3, concerning the city or town and the MNOs available in the area. During the off-peak hours, the recorded values measured were closely matched to the approved indicators. There were few intermittent flaps of throughput and data drop rate, but the measured values were within range.

During the peak period, the test conducted indicated severe drops in data throughput and other indicators. The values in Table 3 , indicates difficulty in accessing the FOB internet service which affirms the work of Karn et al. (2017).

The graph in Fig. 2 indicate drops in data packets between the wireless gateway and the AP; this situation affected the quality of the input signal to the User Equipment (UE). High latency resulted due to greater amount of droped signal power in $\mathrm{dBm}$. Low RSSI measurement puts the performance of the three licensed indicators in poor range below the approved measurable standards (Karn et al., 2017). Three cities experienced major drops in data throughput during the peak and offpeak periods, as shown in graphs in Fig. 2 and 3.

Table 2: Drive-Test Report for off-peak hours

\begin{tabular}{|c|c|c|c|c|}
\hline \multicolumn{2}{|c|}{ Licensed Indicators } & \multirow[b]{2}{*}{$\begin{array}{l}\text { Data Access Success Rate }(\%) \\
(\geq 95 \%)\end{array}$} & \multirow[b]{2}{*}{$\begin{array}{l}\text { Data Drop Rate }(\%) \\
(\leq 1 \%)\end{array}$} & \multirow[b]{2}{*}{$\begin{array}{l}\text { Throughput (Mbps) } \\
\text { ( } \geq 1 \mathrm{Mbps})\end{array}$} \\
\hline Threshold & -------- & & & \\
\hline City & MNO & & & \\
\hline Accra & Net1 & 100 & 0.5 & 160 \\
\hline Accra & Net2 & 100 & 00. & 155 \\
\hline Tema & Net1 & 100 & 0.5 & 150 \\
\hline Tema & Net2 & 100 & 0.5 & 110 \\
\hline Kasoa & Net1 & 100 & 0.5 & 145 \\
\hline Kasoa & Net2 & 100 & 0.5 & 115 \\
\hline Kumasi & Net1 & 100 & 0.0 & 160 \\
\hline Kumasi & Net2 & 100 & 0.0 & 150 \\
\hline Sunyani & Net1 & 100 & 0.0 & 110 \\
\hline Sunyani & Net2 & 100 & 0.5 & 100 \\
\hline
\end{tabular}

Table 3: Drive test report for peak hours

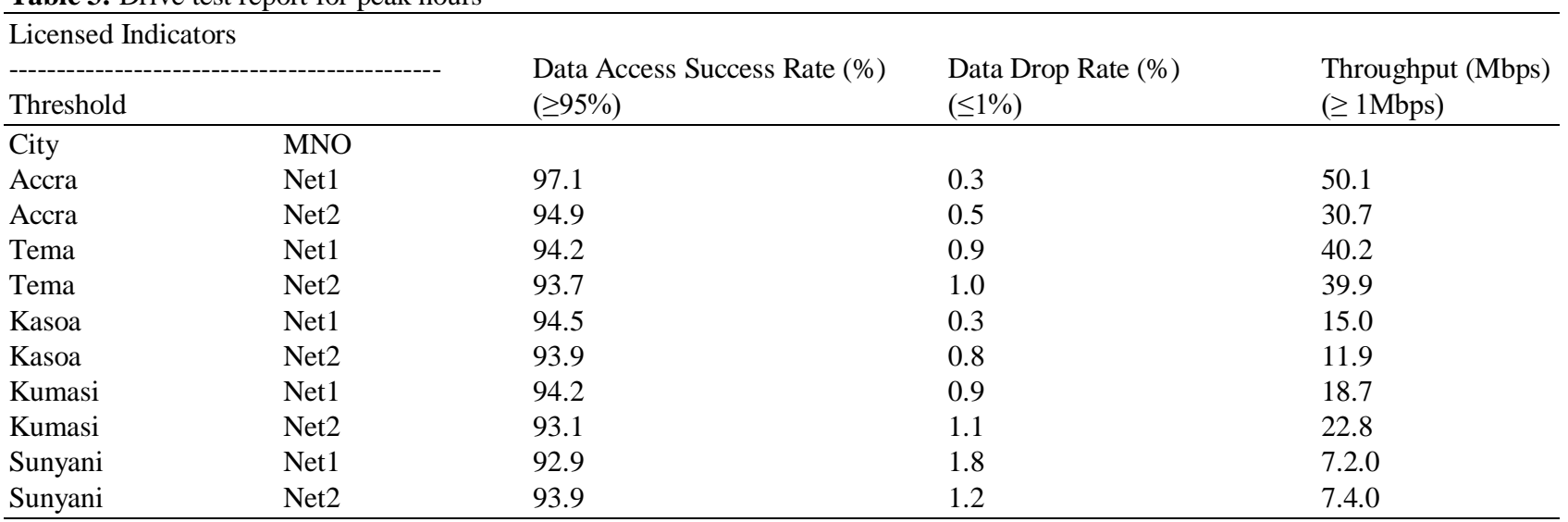




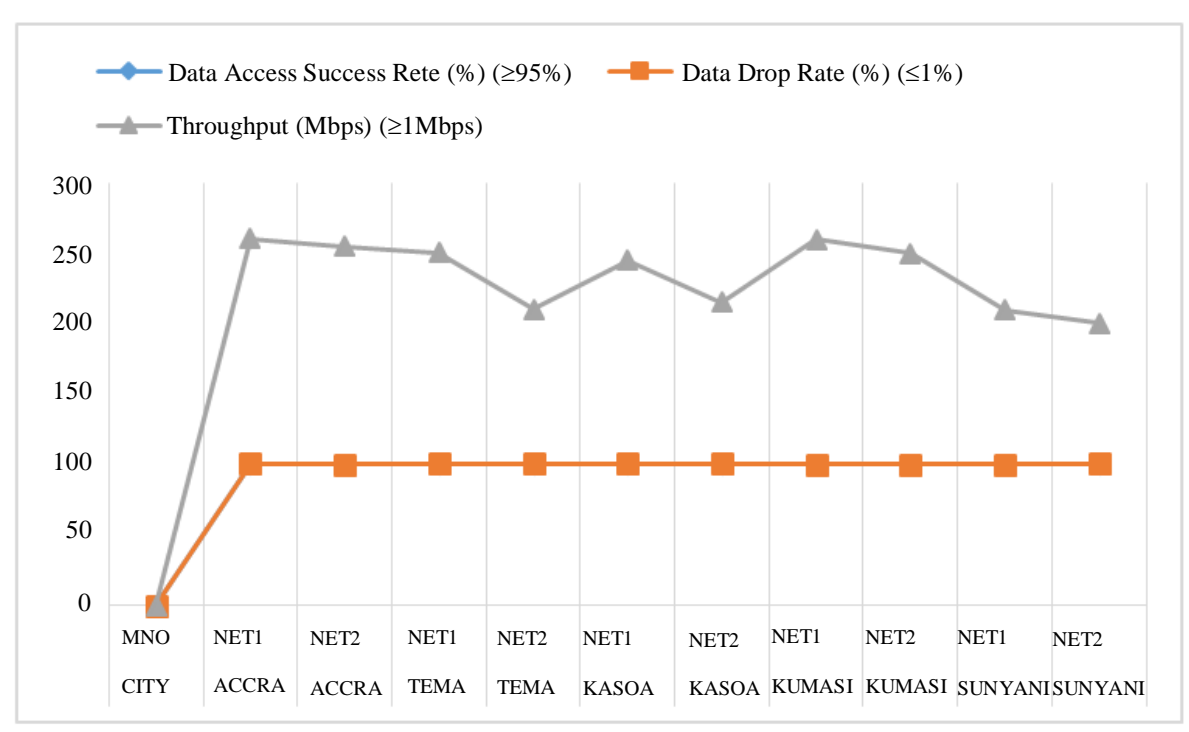

Fig. 2: Off-peak hours

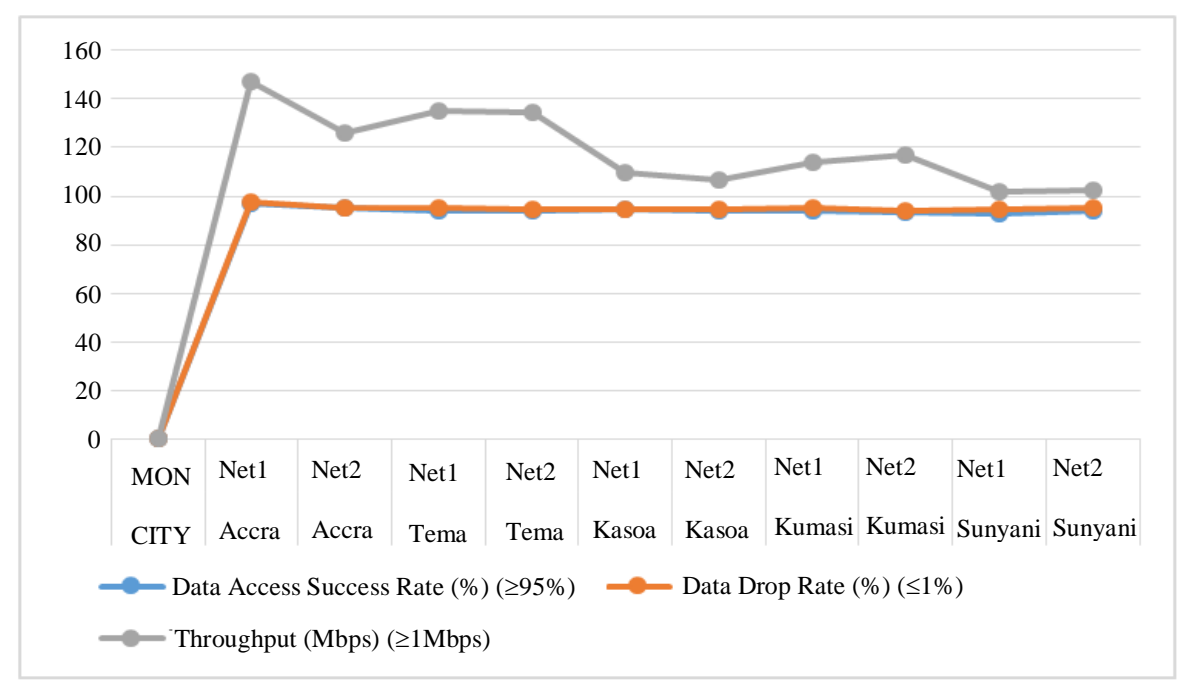

Fig. 3: Peak hours

\section{Proposed Conceptual Framework}

The research sort to measure the performance of the received signal strength which is the determining factor for the adopted three indicators and vice-versa. The strong received signal indicates quality FOB service, thence improved Data Access Success Rate, Data Drop Rate and Data Throughput.

Figure 5 shows the conceptual framework of this paper.

The measured indicators had varied values at different times of the day. The poorly recorded values of the three indicators indicate a weak signal strength due to free space loss and other factors like noise.

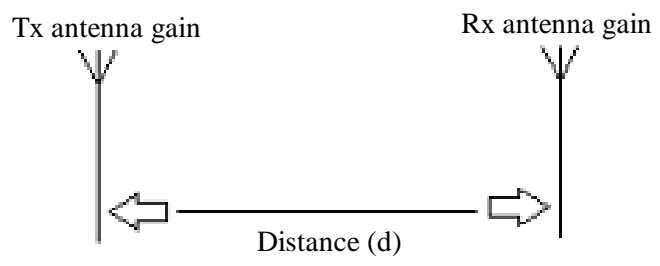

Fig. 4: Free space between the wireless gateway and the AP

Kalman's filter was deployed to improve the signal for distance $d$ as indicated in Fig. 4. The Kalman's technique adopted by the paper because of its ability to filter noise and other factors which weakens signal strength in a free space propagation. 


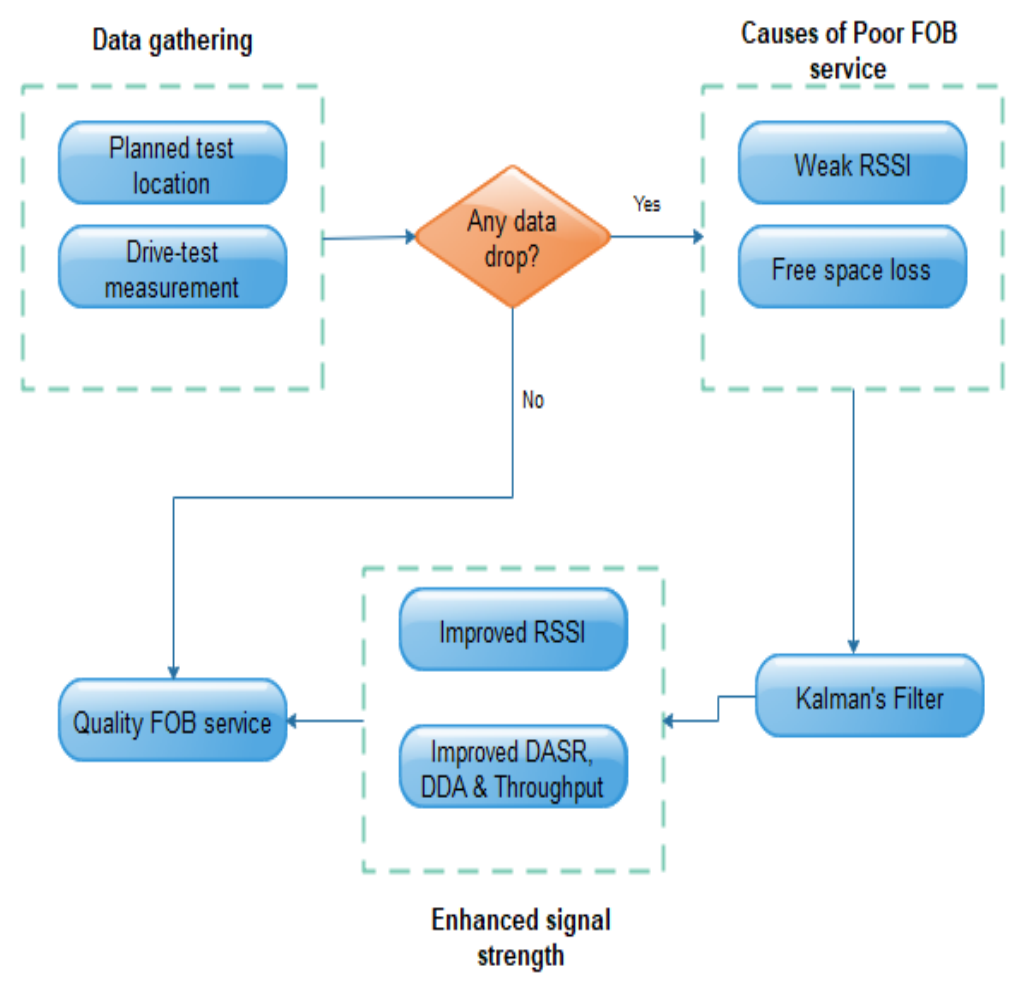

Fig. 5: Conceptual framework of improved RSSI

\section{Received Signal Strength Indicator}

Received Signal Strength Indicator (RSSI) is a measured signal value in wireless networks. It is the signal strength which allows the FOB wireless router to hear from the wireless gateway. The RSSI is measured in decibels $(\mathrm{dBm})$ from 0 (zero) to -100 . The closer the RSSI value to 0 (zero), the stronger the signal and the farther the value is from zero (0), the weaker the signal. Typically voice networks require a $-60 \mathrm{dBm}$ or better signal level while a data network needs $-70 \mathrm{dBm}$ or better. The normal range in a network would be $-45 \mathrm{dBm}$ to $-87 \mathrm{dBm}$ depending on power levels and network design (Han et al., 2018). The Signal is also affected by the wireless router's transmit power and antenna as well as the user's antenna. To improve the performance of RSSI, formula (1) was used:

$$
R S S I \text { in }(d B m)=-10 n \log \frac{d}{d_{0}}+A
$$

where, $n$ is the attenuation factor, parameter $A$ is the offset which is the measured RSSI value at a reference point from the wireless gateway and $d$ is the distance from the wireless router, $\mathrm{d}_{0}$ is the reference distance to be in far field conditions.

However, noise and other factors affects the quality of a broadband signal (Zhang et al., 2016). Noise is any signal that interferes with the actual wireless signal. Noise can be detected from other wireless devices such as cordless phones, microwave devices and the atmospheric particles etc. This value can also be measured in decibels from 0 (zero) to -100 . Noise level is the amount of interference detected in the wireless signal. To achieve low noise in FOB network infratructure, technical measures must be adapted to improve the RSSI value; thus, the lower the noise level, the better the signal strength becomes (Han et al., 2018; $\mathrm{Yu}$ et al., 2014). The reduced noise level improves the signal quality, which lead to an enhanced performance of the three main indicators this paper used to assess the quality of service of fiber optics broadband (Varma, 2015). Kalman's filter was used to achieve this.

\section{Free Space Path Loss}

Free space path loss is the weakening of the radio frequency signal due to the broadening of the signal waveform. It is a measure of how much signal power the device loses over a given distance (Han et al., 2018). Typically, devices lost about $0.020 \mathrm{~dB}$ per foot in an outdoor or wide open space; doors, walls, glass, etc. affect this. This is why the farther the AP is positioned away from the wireless gateway, the weaker the signal becomes (Shaddad et al., 2012).

All this relates to the user because it determines the signal the AP receives, So if the user's AP has a $2 \mathrm{dBi}$ 
antenna, it improves the inbound signal and assuming the actual RSSI signal detected is $-68 \mathrm{dBm}$, then:

$$
\begin{aligned}
& \text { Actual RSSI + Antenna Gain = Displayed } \\
& \text { RSSI-68dBm }+2 \mathrm{dBm}=-66 \mathrm{dBm}
\end{aligned}
$$

Free space path loss can also be estimated using the following formula:

$$
\begin{aligned}
& F S P L=\left(\frac{4 \pi d}{\lambda}\right)^{2} \\
& F S P L=20 \log _{10}(d)+20 \log _{10}(f) \\
& +20 \log _{10}\left(\frac{4 \pi}{c}\right)-G_{T_{x}}-G_{T_{x}}
\end{aligned}
$$

where, $d$ is the distance of the AP from the transmitter, $\lambda$ is the signal wavelength, $f$ is the signal frequency and $c$ is the speed of light in a vacuum, $\mathrm{G}(\mathrm{Tx})=$ The Gain of the Transmitting Antenna and $\mathrm{G}(\mathrm{Rx})=$ The Gain of the Receiving Antenna. Figure 4, show the distance between the wireless gateway and the AP.

\section{The Kalman Filter as a Chi-Square Merit Function for RSSI}

The objective of the adoption of Kalman's filter in this work is to minimize the Mean Squared Error (MSE) between the actual and estimated broadband signal between the wireless gateway and the wireless AP. The minimization of the MSE implies a boost of RSSI measurement. Kalman's filter provides the best prediction of the broadband signal (Han et al., 2018) of mean squared error. That notwithstanding, Kalman's filter has been adopted as the chi-square in this work, to improve the signal strength of the FOB (Shaddad et al., 2012).

The chi-square merit function is a maximum likelihood function and it is typically used as a criterion to fit a set of model parameters to a model a process known as least squares fitting.

The chi-square merit function is:

$$
x^{2}=\sum_{i=1}^{k}\left\lceil\frac{z_{i}-h\left(a_{i}, x\right)}{\sigma_{i}}\right\rceil^{2}
$$

\section{Where:}

$z_{i}=$ The measured value

$h_{i}=$ The data model with parameters $x$, assumed linear in a

$\sigma_{i}=$ The variance associated with the measured value

The optimal set of parameters to be improved can then be defined as that which minimizes the above function. Expanding out the variance gives: $x^{2}=\sum_{i=1}^{k} \frac{1}{\sigma_{i} \sigma_{i}}\left\lceil z_{i}-h\left(a_{i}, x\right)\right\rceil^{2}$

Representing the chi-square in vector form and using notation from the earlier Kalman derivation:

$x_{k}^{2}=\left[z_{k}-h\left(a, x_{k}\right)\right] R^{-1}\left[z_{k}-h\left(a, x_{k}\right)\right]^{T}$

where, $R^{-1}$ is the matrix of inverse squared variances of the signal, i.e., $\frac{1}{\sigma_{i} \sigma_{i}}$.

The above merit function is associated with the latest, kth, measurement and provides a measure of how accurate the model expected RSSI to be improved. Given that the inverse model covariance matrix is known up to time $k$ to receive data from the wireless gateway, the merit function up to time $k$ may be re-written as:

$x_{k-1}^{2}=\left(x_{k-1}-\hat{x}_{k-1}\right) P_{k-1}^{i-1}\left(x_{k-1}-\hat{x}_{k-1}\right)^{T}$

To combine the improved signal with the previous, the fitting signal strength models the parameters so as to minimize the overall chi-square function; the merit function then becomes the summation of the two equations:

$x^{2}=\left(x_{k-1}-\hat{x}_{k-1}\right) P_{k-1}^{i-1}\left(x_{k-1}-\hat{x}_{k-1}\right)^{T}$

$\left[z_{k}-h\left(a, x_{k}\right)\right] R^{-1}\left[z_{k}-h\left(a, x_{k}\right)\right]^{T}$

where, $P_{k-1}^{i-1}$ is the prior estimate of $P_{k} . P_{k}$ is the error covariance matrix at time $k$.

$K_{k}$ is the Kalman gain:

$K_{k}=\left[P_{k}^{i-1}+H^{T} R^{-1} H\right]^{-1} H^{T} R^{-1}$

and:

$x_{k}=\hat{x}_{k}-K_{k}\left[z_{k}-h\left(a, \hat{x}_{k}\right)\right]$

Equation (10) is identical to (11) and describes the improvement of the parameter estimation using the error between measured and model projected values of RSSI.

\section{Mean Squared Error}

The Mean Square Error (MSE) of the RSSI measurement can be given by:

$y_{k}=a_{k}+n_{k}$

Where:

$y_{k}=$ The time-dependent observed signal

$a_{k}=$ A gain term

$x_{k}=$ The information bearing signal

$n_{k}=$ The additive noise 
The overall objective is to estimate $x_{k}$. The fiber optics broadband signal received by the wireless AP has a difference between the estimate of $\hat{x}_{k}$ and $x_{k}$, which is known as the error:

$$
f\left(e_{k}\right)=f\left(x_{k}-\hat{x}_{k}\right)
$$

The estimation of $f\left(e_{k}\right)$ is largely dependent upon the distance between the wireless gateway and the wireless AP and the noise identified in space (Zhang et al., 2016); however, it is also clear that the signal (Yu et al., 2014) should be both good and increase monotonically. An error function which exhibits these characteristics is the squared error function:

$$
f\left(e_{k}\right)=\left(x_{k}-\hat{x}_{k}\right)^{2}
$$

Since it is necessary to consider the ability of the Kalma's filter to minimize the error over a certain distance with a meaningful metric and an expected value of the error function:

$$
\text { loss function }=E\left(f\left(e_{k}\right)\right)
$$

This results in the Mean Squared Error (MSE) function:

$$
\epsilon(t)=E\left(e_{k}^{2}\right)
$$

\section{Maximum Likelihood of Achieving Quality RSSI}

The quality of signal (Zhang et al., 2016) handed over by the wireless gateway to the wireless AP must be improved considerably in time $k$. This can be achieved by redefining the goal of the filter to find $\hat{x}$ which maximizes the probability or likelihood of $y$. That is:

$$
\max [P(y \mid \hat{x})]
$$

Assuming that the additive random noise is Gaussian distributed with a standard deviation of $\sigma_{\mathrm{k}}$ then:

$$
P\left(y_{x} \mid \hat{x}_{k}\right)=k_{k^{\mathrm{exp}}}-\left(\frac{\left(y_{k}-a_{k} \hat{x}_{k}\right)^{2}}{2 \sigma_{k}^{2}}\right)
$$

where, $k_{k}$ is an RSSI normalization constant. The maximum likelihood function of this is:

$$
P\left(y_{x} \mid \hat{x}_{k}\right)=\prod_{k} k_{k} \exp _{-}\left(\frac{\left(y_{k}-a_{k} \hat{x}_{k}\right)^{2}}{2 \sigma_{k}^{2}}\right)
$$

which leads to:

$$
\log P\left(y_{x} \mid \hat{x}_{k}\right)=\frac{1}{2} \sum_{K}\left(\frac{\left(y_{x}-a_{k} \hat{x}_{k}\right)^{2}}{\sigma_{k}^{2}}\right)
$$

The driving function of Equation 20 is the MSE, which may be maximized by the variation of $\hat{x}_{k}$. Therefore the MSE function is applicable when the expected variation of $y_{k}$ is best modelled as a Gaussian distribution. In such a case the MSE serves to provide the value of $\hat{x}_{k}$ which maximizes the likelihood of the signal $y_{k}$. This indicate that the signal strength has been improved with the technique deployed.

\section{Discussion}

Various considerations to improve the RSSI has been critically looked into and an effort to achieve a higher measured RSSI value between the wireless gateway and the AP has been made by computing the expected signal loss using the loss functions and minimize MSE using Kalman's filter and then find a significant antenna gain to compensate for the signal loss. Antenna gain improvement enhances the RSSI measurement as predicted by Kalman's filter. Replacement of smaller-sized antennae with an appropriate size must propagate quality FOB wireless signal over $1.5 \mathrm{~km}$ distance; signal power in $\mathrm{dBm}$ must be increased during the peak hours to control losses and compensate for the signal losses due to the scattering, reflection, diffraction, refraction, fading, etc., of the wireless signal. Belgacem and Lamir (2018) and Khan et al. (2014) used Kalman's filter to improve the signal strength of the wireless network. Kalman's filter has been used by this paper to improve the quality FOB signal strength transmitted between the wireless gateway and the reference distance of the AP. Figure 6 shows the simulation of the improved signal strength $y_{k}$ over a distance $d$ in time $k$ which sort to enhance the three adopted indicators. In order to have stable and quality signal strength, the gain of the wireless gateway antenna was increased to compensate for the free space loss during the peak periods (Shaddad et al., 2012). Increasing the antenna gain and the use of Kalman's filter obviously, brought a significant modification into the FOB network infrastructure. The modified FOB network infrastructure provides better FOB services and overcomes the poor QoS which include but not limited to packet loss, error bit rate, poor throughput, transmission delay, service unavailability, jitter, latency due to limited infrastructure (Boateng and Adekoya, 2019). 


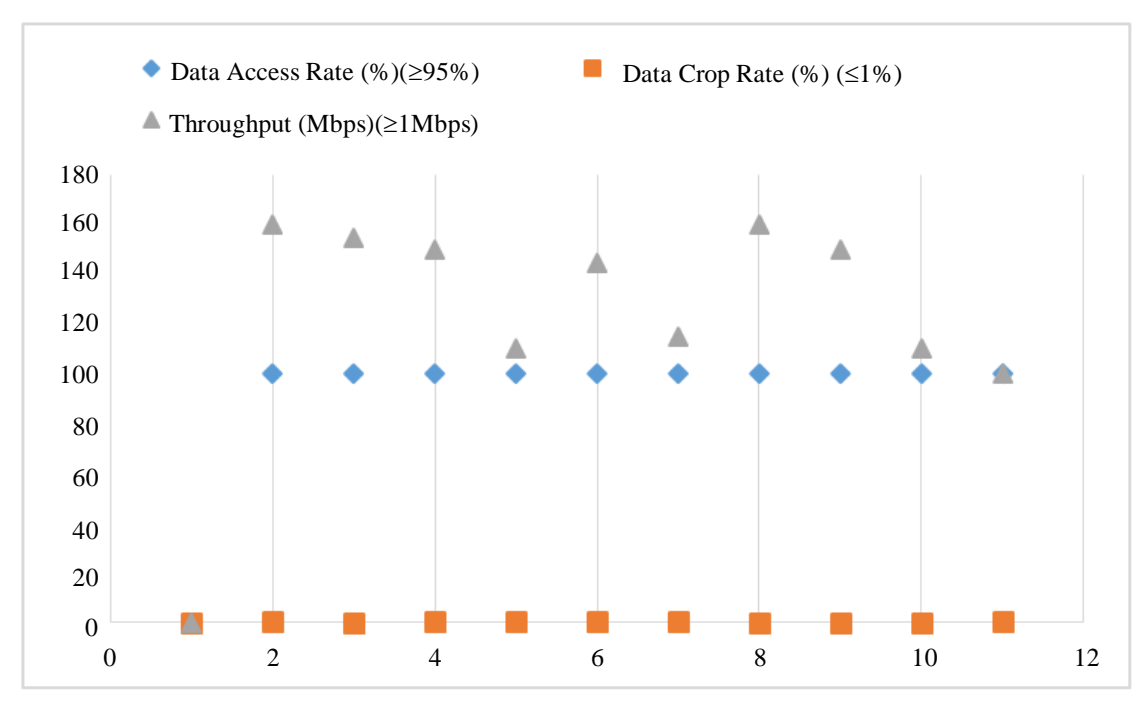

Fig. 6: An improved RSSI to compensate for the licensed indicators

\section{Conclusion}

FOB service is a growing technology which aids users to reach out to their objective and to achieve their business plan. This paper sought to investigate the performance of the FOB service, which has gained so much popularity in the industry, homes and offices of the users. The research examines the three key performance indicators which measures the efficiency and quality of service of broadband services. The test conducted at peak and off-peak periods revealed some drawbacks in the received signals at certain periods over a certain distance. The drops of data packets due to weak signal strength were identified to be severe in most locations at peak periods. Some techniques were used to calculate for the loss signals. In order to improve the RSSI value, Kalman's filter was used as a normalizer to compensate for the loss signal strength. The improved RSSI value saw the three adopted indicator improved significantly, almost matching the measured drive-test values of the off-peak periods. The research has been able to achieve its objective to assess, analyze and then suggest plans to improve the quality of service of the fiber optics broadband service.

\section{Acknowledgement}

We express our profound gratitude to all those whose relentless efforts made this work a success. Those who gave us the drive test device to conduct our signal strength test (they did not want their names to appear in this text). We adopted three regulatory licensed indicators-Data Access Success Rate, Data Drop Rate and Data Throughput from the National Communications Authority (NCA) of Ghana, we are most grateful to them. We also express our thankfulness to the two telecommunications companies we conducted the drive test on their system. We are grateful to all those who reviewed and offered constructive critics and suggestions to improve the work.

\section{Author's Contributions}

All authors contributed immensely to the success of this paper. The corresponding author conducted the drive test, collected the required data and the other authors helped in data analysis, manuscript review, and proposed models to support the work.

\section{Ethics}

There are no known ethical issues that may arise after this paper has been published. We have fully acknowledged all the involved parties, referenced all cited text and equations used. If we have omitted any reference of text or equations cited, we ask the authors to kindly contact the corresponding author of this paper directly to resolve the issue.

\section{References}

Ahmadi, S., 2011. Introduction to Mobile Broadband Wireless Access. In: Mobile WiMAX, Ahmadi, S. (Ed.), Academic Press, ISBN-10: 9780123749642, pp: 1-31.

Belgacem, B. and S. Lamir, 2018. Optimal distributed power control in wireless cellular network based on mixed Kalman/Hoofiltering. AEU - Int. J. Electr. Commun., 90: 103-109.

DOI: 10.1016/j.aeue.2018.04.016 
Bhatt, U.R., A. Dhakad, N. Chouhan and R. Upadhyay, 2019. Fiber wireless (FiWi) access network: ONU placement and reduction in average communication distance using whale optimization algorithm. Heliyon, 5: e01311-e01311. DOI: 10.1016/J.HELIYON.2019.E01311.

Boateng, K., A. Acakpovi, I. Hanson and O. NyarkoBoateng, 2019. Evaluation and analysis of the major security systems deployed on telecommunications cell sites. Int. J. Sci. Eng. Res., 10: 770-777.

Ghassemlooy, Z., S. Zvanovec, M.A. Khalighi, W.O. Popoola and J. Perez, 2017. Optical wireless communication systems. Optik, 151: 1-6. DOI: 10.1016/J.IJLEO.2017.11.052

Hadi, M.U., H. Jung, S. Ghaffar, P.A. Traverso and G. Tartarini, 2019. Optimized digital radio over fiber system for medium range communication. Opt. Commun., 443: 177-185.

DOI: 10.1016/j.optcom.2019.03.037

Han, P., Y. Liu and L. Guo, 2018. QoS satisfaction aware and network reconfiguration enabled resource allocation for virtual network embedding in Fiberwireless access network. Comput. Netw., 143: 30-48. DOI: 10.1016/J.COMNET.2018.06.019.

Kakati, D. and S.C. Arya, 2018. A full-duplex optical fiber/wireless coherent communication system with digital signal processing at the receiver. Optik, 171: 190-199. DOI: 10.1016/J.IJLEO.2018.05.140

Karn, N.K., Z. Hongli and M. Shafiq, 2017. Measuring broadband internet performance in Nepal: A comparative study. Proc. Comput. Sci., 107: 64-69. DOI: 10.1016/J.PROCS.2017.03.057
Khan, R., S.U. Khan, S. Khan and M.U.A. Khan, 2014. Localization performance evaluation of extended kalman filter in wireless sensors network. Proc. Comput. Sci., 32: 117-124. DOI: $10.1016 /$ j.procs.2014.05.405

Nyarko-Boateng, O. and A.F. Adekoya, 2019. Evaluation and analysis of key performance indicators which affect QoS of mobile call traffic. Int. J. Comput. Netw.

Shaddad, R.Q., A.B. Mohammad and A.M. Al-hetar, 2012. Spectral efficient hybrid Wireless Optical Broadband Access Network (WOBAN) based on transmission of wireless MIMO OFDM signals over WDM PON. Opt. Commun., 285: 4059-4067. DOI: 10.1016/J.OPTCOM.2012.06.002

Varma, S., 2015. Congestion control in broadband wireless networks. Internet Congest. Control, 5: 103-134. DOI: 10.1016/b978-0-12-803583-2.00004-9

Yu, Y., Y. Liu, Y. Zhou and P. Han, 2014. Planning of survivable cloud-integrated Wireless-optical broadband access network against distribution fiber failure. Optical Switch. Netw., 14: 217-225. DOI: 10.1016/J.OSN.2014.05.014

Zhang, X., T. Liu and D. Shen, 2016. Investigation of broadband digital predistortion for broadband radio over fiber transmission systems, Opt. Commun., 381: 346-351. DOI: 10.1016/J.OPTCOM.2016.07.025 